\title{
$\alpha$-Tertiary Dialkyl Ether Synthesis via Reductive Photocatalytic $\alpha-$ Functionalization of Alkyl Enol Ethers
}

\author{
Jamie A. Leitch, Thomas Rossolini, Tatiana Rogova, Darren J. Dixon* \\ Department of Chemistry, University of Oxford, Chemistry Research Laboratory, 12 Mansfield Road, Oxford OX1 \\ 3TA, United Kingdom \\ KEYWORDS ether, photoredox catalysis, reductive, radicals, quaternary center
}

\begin{abstract}
The photocatalytic construction of $\mathrm{C}\left(\mathrm{sp}^{3}\right)$-rich $\alpha$-tertiary dialkyl ethers through the reductive $\alpha$ functionalization of alkyl enol ether substrates with conjugated alkenes in the presence of a Hantzsch ester terminal reductant under blue LED irradiation, is described. Pivoting on oxocarbenium ion generation via an initial TMSClfacilitated protic activation of the enol ether substrate, subsequent single electron transfer delivers the key nucleophilic $\alpha$ oxy tertiary radical capable of productively combining with a variety of alkene substrates. The new reductive functionalization strategy was simple to perform, efficient, broad in scope with respect to both alkene acceptor and enol ether donor fragments and delivered a wide range of complex $\alpha$-tertiary dialkyl ether architectures.
\end{abstract}

Dialkyl ethers are commonplace structural motifs within biologically relevant molecules, including active pharmaceutical agents and natural products. ${ }^{1}$ Accordingly, their synthesis - from the traditional Williamson ether synthesis $^{2}$ to recent developments in $\mathrm{C}-\mathrm{H}$ functionalization ${ }^{3}-$ has remained a common focus for classical and contemporary method development. Despite advances in recent years, synthetic access to sterically hindered dialkyl ethers remains a significant challenge, especially for the generation of $\alpha$-tertiary dialkyl ethers, which are becoming increasingly desirable structures in drug design due to favorable metabolic profiles. ${ }^{4}$ To this end, pioneering investigations from the Baran ${ }^{5}$ and Ohmiya ${ }^{6,7}$ groups have exploited decarboxylative single-electron pathways to access reactive carbocation intermediates from $\alpha$-tertiary carboxylic acids and redox active ester derivatives (via electrochemical and photochemical means, respectively) which upon interception by alcohol nucleophiles yield the target dialkyl ether structures (Scheme $1 \mathrm{~A}$ ).

As a complementary strategy, we envisaged that the dialkyl $\alpha$-oxy radical could be leveraged into creating such $\alpha$ tertiary dialkyl structures (Scheme 1B). Previous contributions from the Doyle ${ }^{8}$ and Wang9 groups, established reactivity employing nickel catalysis using a selection of aldehyde-derived acetals creating analogous $\alpha$-secondary ethers. Our own photocatalytic studies ${ }^{10}$ demonstrated efficiency with aryl substituted ketals (blue $=$ Ar), however were largely unsuccessful when applied to dialkyl ketals. Furthermore, due to their synthetic origin, the above methods are entirely limited to small alkyl chains on the oxygen atom (purple)."

Notwithstanding the established polarity of enol ethers in two-electron reaction pathways (nucleophilic at the $\beta$ position, ${ }^{12}$ and, following protonation, electrophilic at the $\alpha$-position ${ }^{13}$ ), developments in redox catalysis ${ }^{14}$ led by Baran and Shenvi have demonstrated that via the $\alpha$-oxy radical, the polarity of the enol ether functionality can be reversed. ${ }^{15}$ However, despite the breadth of chemical space accessible using the established metal-based hydrogen atom transfer (HAT) approach, $\alpha$-tertiary dialkyl ethers remain out of reach.

Scheme 1. $\alpha$-tertiary dialkyl ether synthesis in context of this work

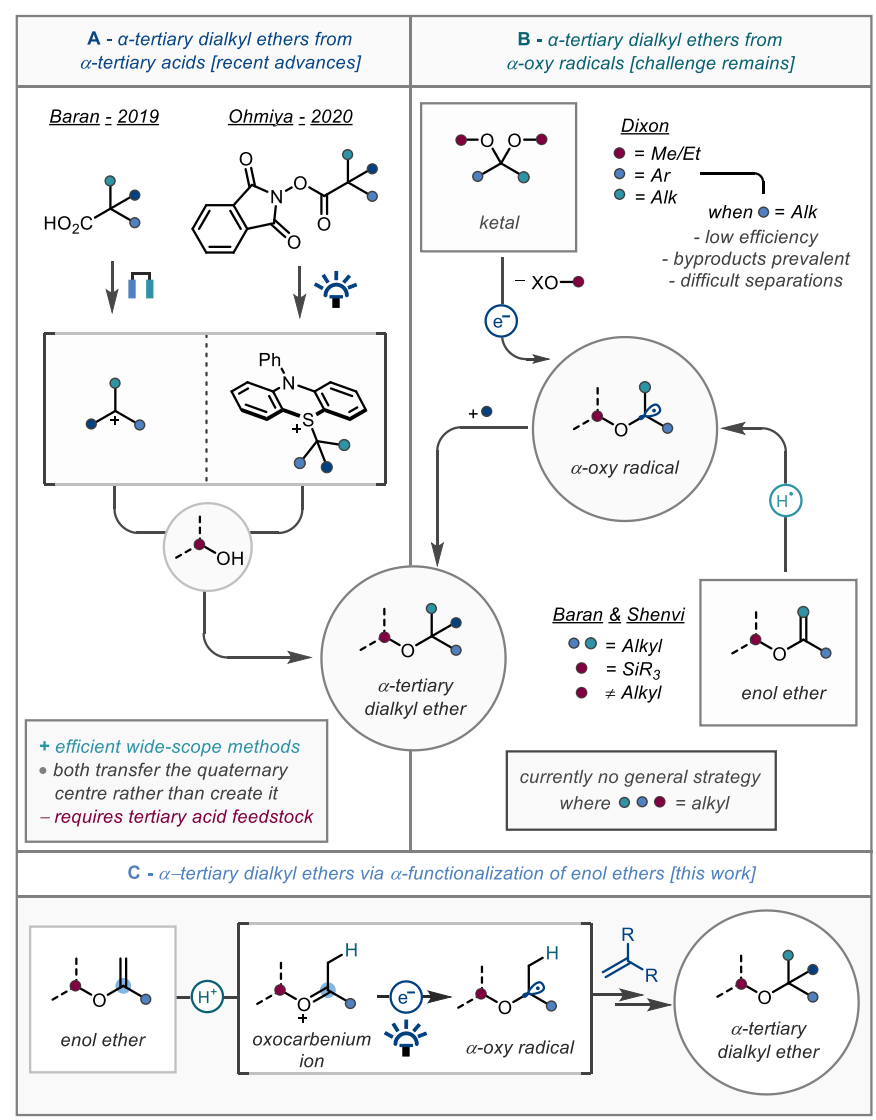


In such cases, the propensity for competitive oxocarbenium formation in the alcohol containing media hindered product formation when similar silyl enol ether derivatives were employed, a phenomenon even more pronounced in all $\mathrm{C}\left(\mathrm{sp}^{3}\right)$-substituted enol ethers. ${ }^{14 \mathrm{~b}}$

Despite the above advances, to date there is no general strategy to construct all-alkyl substituted $\alpha$-tertiary ethers via the $\alpha$-oxy radical. As part of our ongoing work into the reductive synthesis of ethers, ${ }^{16}$ we reasoned that controlled, photocatalytic single electron transfer to the oxocarbenium ion formed via $\beta$-protonation of the enol ether could provide a complementary access point to the $\alpha$-oxy radical. ${ }^{17}$ These nucleophilic radical species would then be reactive towards electrophilic structures such as conjugated alkenes. ${ }^{18}$ As enol ethers are abundant commodity chemicals, or readily accessed via alcohol isopropenylation $^{19}$ or ester methylenation, ${ }^{20}$ this approach could offer a general method for $\alpha$-tertiary dialkyl ether synthesis, and herein we wish to report our findings.

For a model system we chose feedstock 2methoxypropene (1a) as the alkyl enol ether substrate and a dehydroalanine derivative (2a) as the acceptor alkene. Initial reductive coupling experiments were performed using $\left[\operatorname{Ir}\left(\mathrm{dFCF}_{3}(\mathrm{ppy})\right)_{2}(\mathrm{dtbbpy})\right] \mathrm{PF}_{6}$ as photocatalyst, the phenyl-substituted Hantzsch ester derivative (HE-Ph) as the terminal reductant, in DMF as solvent, under blue light irradiation (Table 1 ). In the absence of a Brønsted or Lewis acid additive, no reactivity was observed towards a $\mathrm{C}-\mathrm{C}$ coupled product (3a). On addition of stoichiometric Brønsted acid additive para-toluenesulfonic acid (entry 2), conversion to an alternative ester addition product zaa was observed. This structure is proposed to form via a Hantzsch ester degradation pathway (see supporting information for more details). Use of stoichiometric triflic acid as a stronger Brønsted acid promoter indeed formed the desired product in $23 \%$ conversion, albeit again coupled with competitive formation of zaa (entry 5). Interestingly however, when TMSOTf was employed as additive without an additional exogenous proton source, moderate reactivity towards 3 a was again observed $(20 \%$ yield, entry 6). The use of trimethylsilyl chloride gave a substantial uplift to reaction efficiency, creating the $\alpha$ tertiary alkyl ether $3 a$ in decent yield (58\%) when 1 equivalent was deployed (entry 7 ), notably in $48 \%$ yield with 30 mol\% of the additive (entry 8 ), and in good yield $(78 \%$ ) with 2 equivalents (entry 9). Furthermore, TBSCl $(52 \%)$ and $\mathrm{TMSBr}(20 \%)$ also enabled the reaction to proceed (entries 10-11). ${ }^{21}$ Despite attaining good yields of the desired product in entry 9, both za and zaa possessed identical polarity when attempting purification using standard chromatographic techniques. Accordingly, continued optimization of the reaction was required to suppress byproduct formation. Exchange of the limiting reagent from the feedstock 2-methoxypropene to the synthesized DHA derivative - readily achieved this providing a yield of $95 \%$ of $3 \mathbf{a}$, and only trace of the glutamate adduct $3 \mathbf{a a}$, denoting a product selectivity of >20:1 (entry 14). Importantly, control experiments without photocatalyst,
Hantzsch ester, or light irradiation gave no conversion to the $\alpha$-tertiary ether product (entries 15-17). ${ }^{22}$

Table 1. Optimization of the $\alpha$-functionalization of alkyl enol ethers
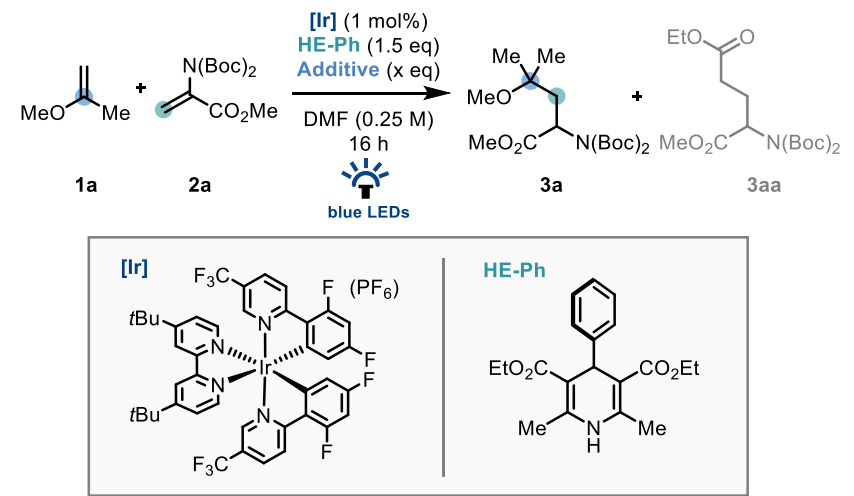

\begin{tabular}{|c|c|c|c|c|c|c|}
\hline entry & Additive ( $x$ eq) & 1a (eq) & $2 a(e q)$ & $3 a^{a}$ & $3 a^{a}$ & $3 a: 3 a^{a}$ \\
\hline 1 & - & 1 & 2 & - & - & $n / a$ \\
\hline 2 & $p \mathrm{TSA}$ (1) & 1 & 2 & - & 56 & $n / a$ \\
\hline 3 & PPTS (1) & 1 & 2 & - & - & $\mathrm{n} / \mathrm{a}$ \\
\hline 4 & PPTS $(1)^{b}$ & 1 & 2 & 5 & 5 & $1: 1$ \\
\hline 5 & TfOH (1) & 1 & 2 & 23 & 27 & $0.9: 1$ \\
\hline 6 & TMSOTf (1) & 1 & 2 & 24 & 29 & $0.8: 1$ \\
\hline 7 & TMSCI (1) & 1 & 2 & 63 & 20 & $2.5: 1$ \\
\hline 8 & TMSCI (0.3) & 1 & 2 & 48 & 25 & $1.9: 1$ \\
\hline 9 & TMSCI (2) & 1 & 2 & 78 & 20 & $3.9: 1$ \\
\hline 10 & TMSBr (2) & 1 & 2 & 20 & 16 & $1.3: 1$ \\
\hline 11 & TBSCI (2) & 1 & 2 & 52 & 22 & $2.4: 1$ \\
\hline 12 & TMSCI (2) & 1.5 & 1 & 79 & 5 & $16: 1$ \\
\hline 13 & TMSCI (2) & 2 & 1 & 78 & 5 & $16: 1$ \\
\hline 14 & TMSCI (2) & 3 & 1 & $95(95)^{c}$ & 1 & $>20: 1$ \\
\hline 15 & \multirow{3}{*}{\multicolumn{3}{|c|}{$\begin{array}{c}\text { no iridium photocatalyst } \\
\text { no Hantzsch Ester } \\
\text { no light }\end{array}$}} & - & - & $n / a$ \\
\hline 16 & & & & - & - & $\mathrm{n} / \mathrm{a}$ \\
\hline 17 & & & & - & - & $n / a$ \\
\hline
\end{tabular}

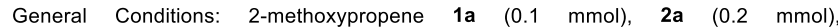
$\left[\operatorname{lr}\left(\left(\mathrm{dFCF}_{3}\right) \mathrm{ppy}_{2}(\mathrm{dtbbpy})\right] \mathrm{PF}_{6}(0.001 \mathrm{mmol}, 1 \mathrm{~mol} \%)\right.$, phenyl Hantzsch ester $(0.15 \mathrm{mmol})$, DMF $(0.25 \mathrm{M}), 16 \mathrm{~h}$, under a nitrogen atmopshere under blue light irradiation using $18 \mathrm{~W}$ LED lamp. ${ }^{a}$ Formation of $\mathbf{3 a}$ and $3 \mathbf{a a}$ calculated by ${ }^{1} \mathrm{H}$ NMR analysis of the crude reaction mixture against $1,3,5$-trimethoxybenzene as an internal standard. ${ }^{b}$ DMA was used as solvent. ${ }^{c}$ Isolated yield after silica gel column chromatography.

With optimal reaction conditions in hand, the scope of our method with respect to the $\alpha, \beta$-unsaturated ester component, using both 2-methoxypropene (1a) and 2benzyloxypropene (1b), was investigated (Scheme $2 \mathrm{~A}$ ). The reaction proceeded with good yields when $\alpha$-substituted acrylate esters were used as the alkene substrate (3c-3g). Furthermore, desirable reactivity was also achieved with a vinyl sulfone acceptor (3h), 1,1-diphenylethylene (3i-3j), and even a vinyl boronate derivative (3l), albeit with reduced yield. Commercially available methyl 2acetamidoacrylate was amenable to the reaction conditions in comparable yields to the model DHA substrate (3b). Using dipeptide-derived DHA analogues, coupling of the 2-methoxypropyl fragment to proline-, 3-pyrrolidine carboxylic acid-, and nipecotic acid-derived alkene derivatives took place in good yields (3m-3o). We also studied a dehydroalanine-derived substrate (2p), which has found previous use as a chiral building block in addition reac- 
tions. ${ }^{23}$ Using the standard protocol, it was shown to forge the $\mathrm{C}-\mathrm{C}$ bond in excellent yield and diastereoselectivity (3p, $82 \%$ yield, $>20: 1 \mathrm{dr}$ ).

The scope with respect to the alkyl enol ether fragment was then studied (Scheme 2B). Substituted benzyloxypropene substrates were effective coupling partners leading to good yields of the $\alpha$-tertiary ether architectures with electron-donating groups and electron-withdrawing groups functioning similarly $(\mathbf{4} \mathbf{b}-\mathbf{4 g})$. Pleasingly, we observed that a cyclobutane-substituted construct was applicable to our enol ether functionalization platform, creating a structurally complex $\alpha$-tertiary alkyl ether in good yield $(\mathbf{4} \mathbf{h})$. A range of other alkoxypropene derivatives were also productive in this methodology forming further oxygenated leucine derivatives (4i-4j). Prolinol- and tetrahydrofurfuryl alcohol-derived enol ethers were also effective substrates (4k-4l).

Scheme 2. Scope of the photocatalytic $\alpha$-functionalization of alkyl enol ethers.
A furanyl-substituted enol ether motif was also amenable to this methodology, with no undesired reactivity observed on the heteroaromatic ring $(\mathbf{4 m})$. 2Alkoxycyclopentene and cycloheptene derivatives were well-tolerated giving the dialkyl ether materials in good to excellent yields (4n, 4p). Surprisingly, the cyclohexyl analogue was a comparatively poor substrate delivering the ether products 40 in trace conversion. Despite this, using exocyclic enol ether 2-methylidenetetrahydropyran (1q) where the oxygen atom is located inside the 6 -membered ring - re-installed reactivity affording (4q) in moderate yield. Finally, this mild photocatalytic protocol was shown to be compatible with acetal-protected ketones. Competitive acetal functionalization was not observed and the desired complex $\alpha$-tertiary dialkyl ether architectures $(\mathbf{4} \mathbf{r}-\mathbf{4} \mathbf{s})$ were obtained in good yields. ${ }^{10}$

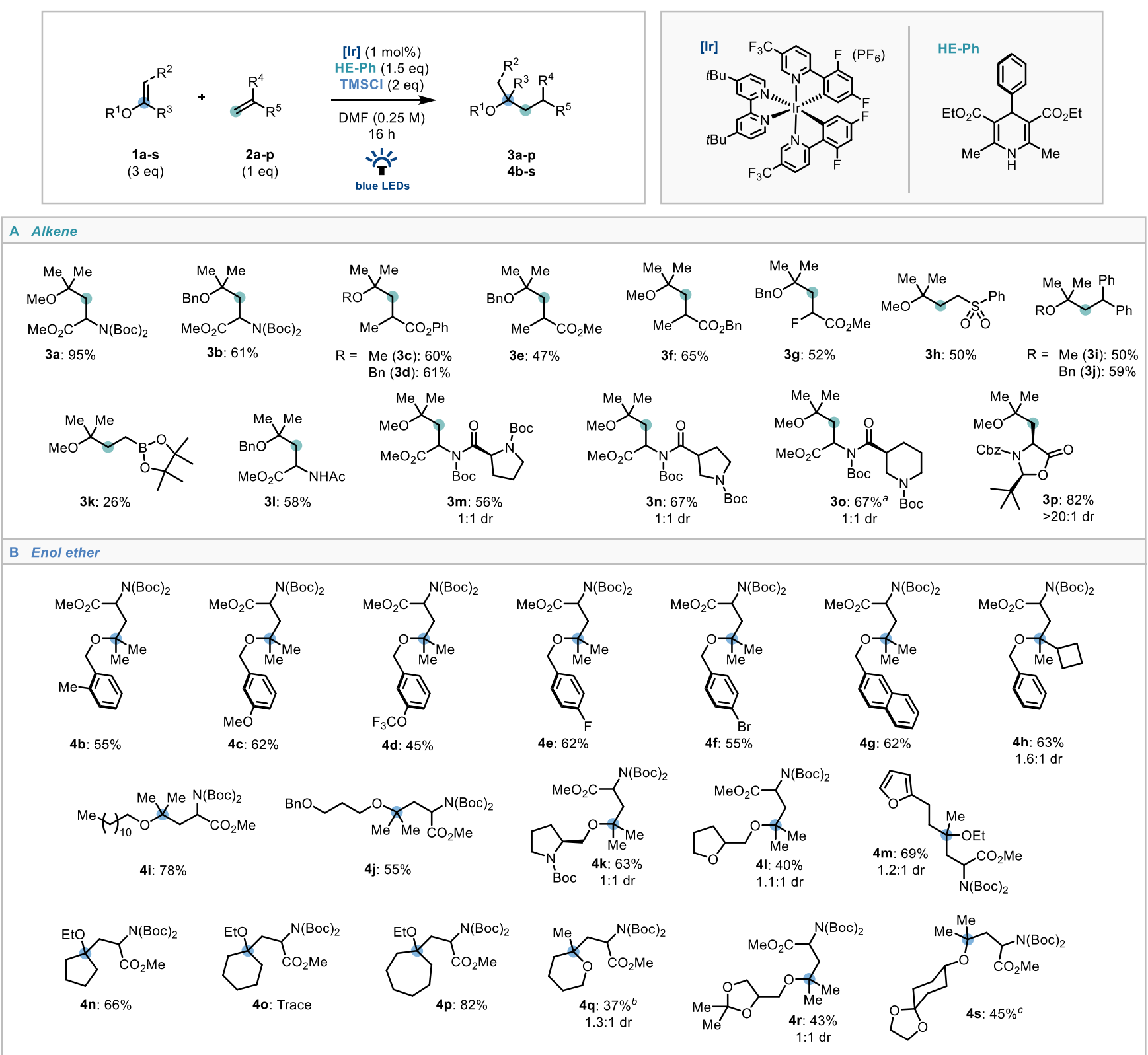

${ }^{a} \mathrm{TMSCl}\left(3\right.$ eq) used. $^{b}$ Enol ether $\left(5\right.$ eq) was used. ${ }^{c} \mathrm{TMSCl}(4$ eq) was used. 
From a mechanistic viewpoint, how the enol ether substrate engages in the reaction pathway to generate the key radical was of interest. Our working hypothesis was that Brønsted acid activation to form the oxocarbenium ion was required prior to electron transfer, and therefore we chose to conduct experiments with deuterium labelled Hantzsch esters and monitor deuterium incorporation in the $\alpha$-tertiary dalkyl ether product (Scheme 3, for further details, see ESI).

In the first of these studies using HE-Ph-d, we observed substantial deuterium incorporation in the diastereotopic $\beta$ and $\beta$ '-position of the product, suggesting Brønsted acidity arises from the $\mathrm{N}-\mathrm{D}$ bond (Scheme $3 \mathrm{~A}$ ). Interestingly

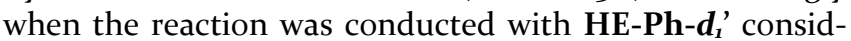
erable deuterium incorporation at the $\gamma$-position was not observed, instead, deuterium labelling was observed around multiple other positions in the product (Scheme ${ }_{3 B} \mathrm{~B} .{ }^{24}$ This result suggests that the proton at the $\gamma$-position in the products arises from an intramolecular $1,4^{-}$or $1,5^{-}$ HAT to the $\gamma$-amino radical intermediate and the resulting alkyl or oxy radical then undergoes intermolecular HAT from the Hantzsch ester or an oxidized derivative. Fur-

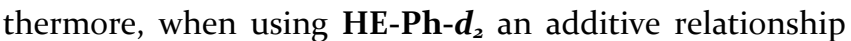
was observed, again with negligible deuterium incorporation at the $\gamma$-position, reinforcing this alternative termination pathway hypothesis (Scheme ${ }_{3} \mathrm{C}$ ). From these studies, we concluded that the $\mathrm{N}-\mathrm{H}$ bond is a source of $\mathrm{H}^{+}$in the activation of the enol ether, and the $\mathrm{C}-\mathrm{H}$ bond is a source of $\mathrm{H} \bullet$ in a HAT event.

Next, we computationally analyzed $\mathrm{p} K_{a}$ values to identify the Brønsted acid activator. $\mathrm{p} K_{a}$ calculations predict that the $\mathrm{HE}$ radical cation (Scheme $3 \mathrm{D}, \mathrm{p} K_{a(\mathrm{DMSO})}=3.0$ ) and Hantzsch pyridinium $\left(\mathrm{p} K_{a(\mathrm{DMSO})}=0.6\right)$ are substantially more acidic than the parent dihydropyridine $\left(\mathrm{p} K_{a}=19 \cdot 7\right)$. ${ }^{1} \mathrm{H}$ NMR experiments also revealed no visible interaction between the $\mathrm{HE}$ and TMSCl in the ground state. When compared to para-toluenepyridinium sulfonate (PPTS), ${ }^{25}$ a Brønsted acid used in alcohol protections using 2methoxypropene, these protic intermediates are comparably acidic. Despite this, the reaction does not proceed in the absence of any additive. We conducted further additive studies to explore the key role of the TMSCl in producing the $\mathrm{C}-\mathrm{C}$ coupled product $3 \mathbf{a}$ (Scheme ${ }_{3} \mathrm{E}$ ). These studies demonstrated that halosilane additives were all effective in forming the $\alpha$-tertiary dialkyl ether motif, with a decreasing trend in reactivity with increasing steric bulk of the silane. Conversely other chloride salts $\left(\mathrm{NH}_{4} \mathrm{Cl}, \mathrm{LiCl}\right.$, $\mathrm{NBu}_{4} \mathrm{Cl}$ ) were substantially inferior or ineffective. These results along with the noticeable difference in reactivity between TMSCl and TMSBr led us to conclude that both the silane and the halide counter ion are of importance to the reaction mechanism. Combining these mechanistic investigations, we propose two potential roles of the chlorosilane additive. Firstly, as a promoter in the protic acid activation of the enol ether, ${ }^{26}$ where one of oxidized forms of the Hantzsch ester is the Brønsted acid and the TMSCl is the Lewis acid. Secondly, as a source of the nucleophilic counter ion which could stabilize such a resulting oxocarbenium intermediate. ${ }^{27}$
Building on these data and previous work, ${ }^{15 a, 28}$ a plausible mechanism is shown in Scheme 4. Initially, the Hantzsch ester derivative $\left(E_{1 / 2}^{\circ}\right.$ (calc) $\left.=+0.75 \mathrm{~V}\right)$ readily quenches the photoexcited iridium(III) species $\left(E_{1 / 2}^{\circ}=+1.21 \mathrm{~V}\right)^{29}$ to deliver the reducing iridium(II) species and the Hantzsch ester radical cation. $3^{\circ}$ Concurrent protic activation of the enol ether takes place to afford the key oxocarbenium ion.

Scheme 3. Deuterium incorporation, $\mathrm{p} K_{a}$ studies, additive effects

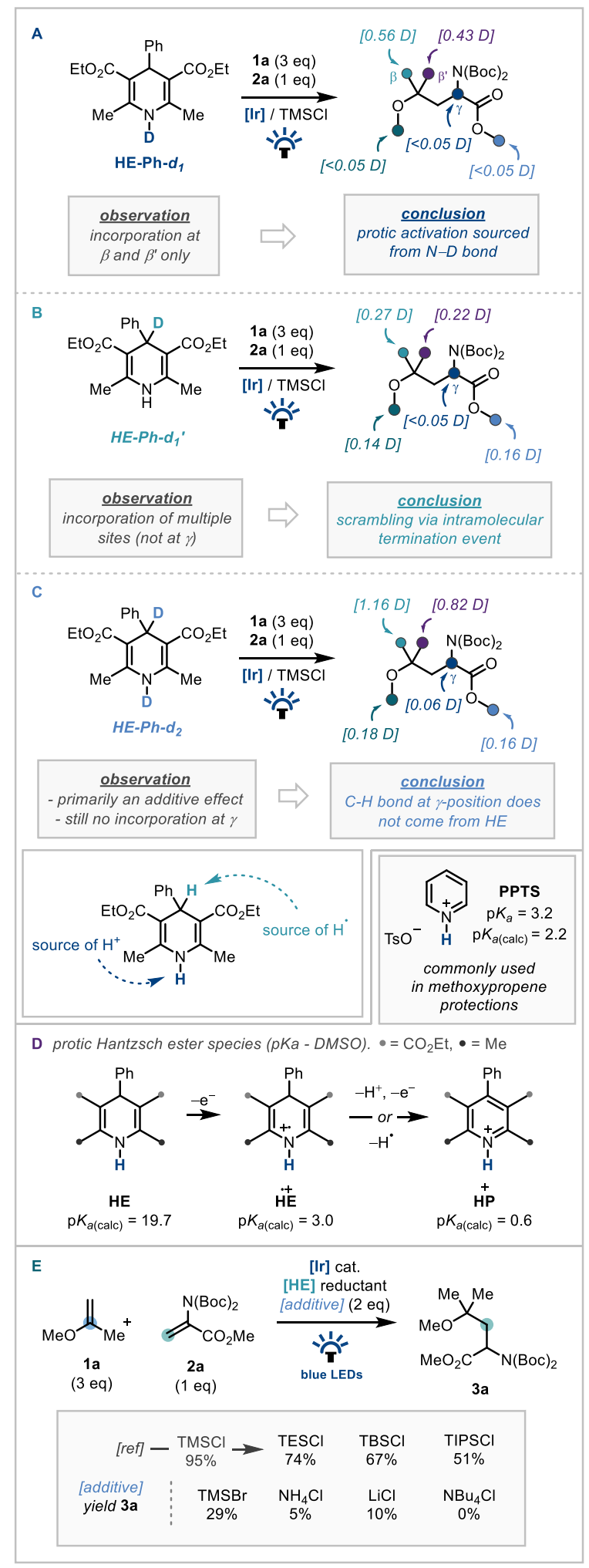




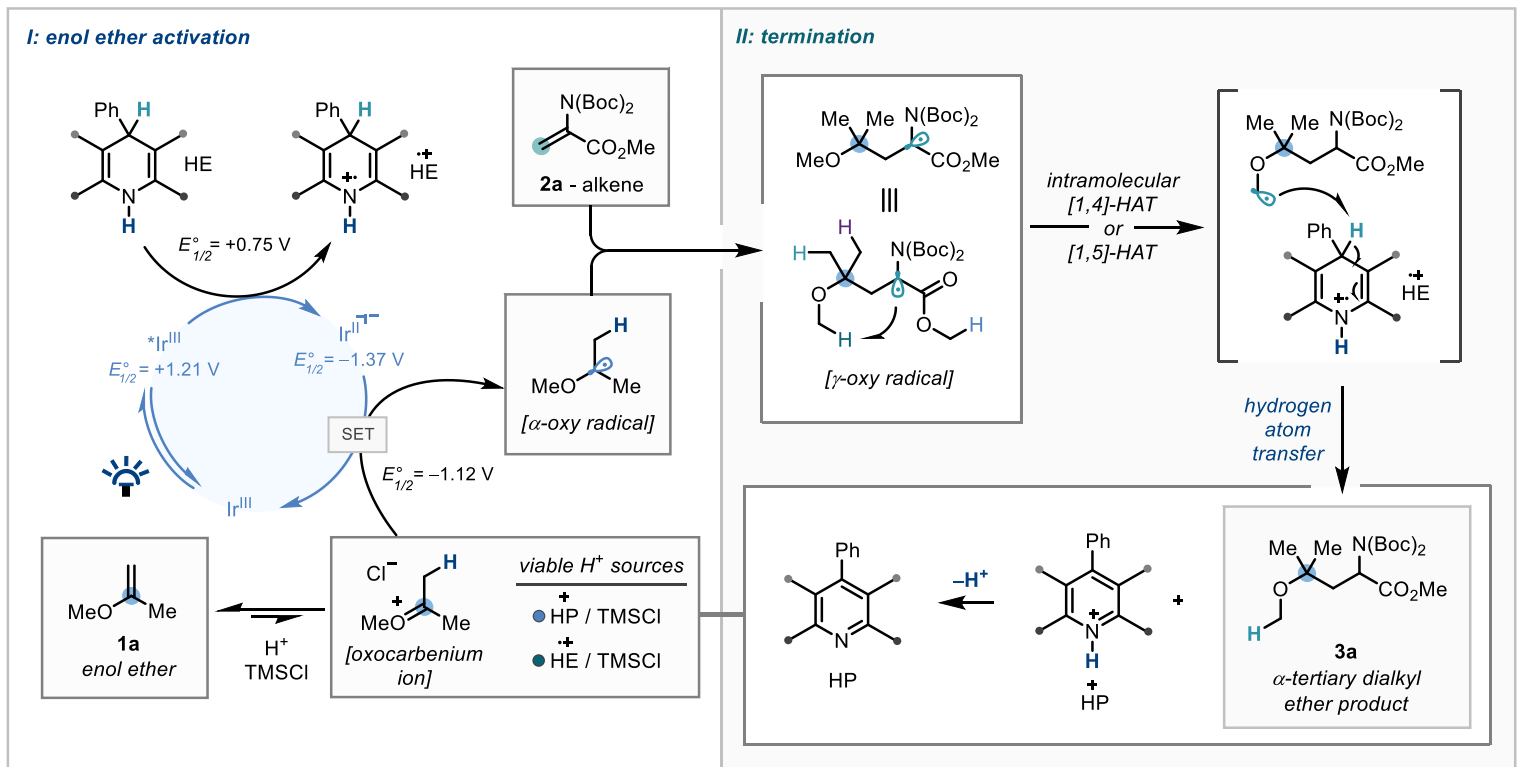

Single electron transfer from the iridium(II) species $\left(E_{1 / 2}^{\circ}\right.$ $=-1.37 \mathrm{~V})$ to the oxocarbenium ion $\left(E_{1 / 2}^{\circ}\right.$ (calc) $\left.=-1.12 \mathrm{~V}\right)$ gives rise to the nucleophilic $\alpha$-oxy radical ${ }^{11}$ which then undergoes Giese-type addition with the conjugated alkene to produce the $\gamma$-amino radical. Subsequent intramolecular HAT and then intermolecular HAT permits termination, delivering the $\alpha$-tertiary dialkyl ether 3 a. This HAT can take place from either HE radical cation (shown, Scheme 4, right), or $\mathrm{HEH} \cdot$, rendering either $\mathrm{HP}+$ or $\mathrm{HE}$ radical cation as the potential respective proton sources for the corresponding mechanisms (see ESI for more details). Aligned to previous investigations, the pathway shown is most likely in operation..$^{32}$

In conclusion, a mild photocatalytic reductive $\alpha$ functionalization of alkyl enol ethers has been developed. Through Lewis acid-assisted protic activation of the enol ether to generate the corresponding oxocarbenium ion and subsequent single electron reduction, we have identified a new approach to access the $\alpha$-oxy dialkyl radical. This reactive intermediate was shown to engage in Giesetype coupling reactions with a wide range of alkene substrates to deliver 33 examples of $\alpha$-tertiary dialkyl ether architectures. Work to understand the source of the acidity and applying this concept to further reaction systems is currently underway.

\section{AUTHOR INFORMATION}

\section{Corresponding Author}

*darren.dixon@chem.ox.ac.uk

\section{ACKNOWLEDGMENT}

J.A.L. thanks the Leverhulme Trust (RPG-2017-069) for a research fellowship. T.Rossolini thanks Astex Pharmaceuticals, for financial support. T.Rogova is supported by the EPSRC Centre for Doctoral Training in Synthesis for Biology and Medicine (EP/Lo15838/1), generously supported by
AstraZeneca, Diamond Light Source, Defence Science and Technology Laboratory, Evotec, GlaxoSmithKline, Janssen, Novartis, Pfizer, Syngenta, Takeda, UCB, and Vertex. T. R. also thanks the Royal Commission of 1851 Industrial Fellowship and NSERC PGS-D. The authors thank Daniel Matheau-Raven for insightful discussions.

\section{REFERENCES}

(1) (a) McGrath, N. A.; Brihacek, M.; Njardarson, J. T. A Graphical Journey of Innovative Organic Architectures That Have Improved Our Lives. J. Chem. Ed. 2010, 87, 1348-1349; (b) McKeage, K.; Keam, S. J. Salmeterol/fluticasone propionate: a review of its use in asthma. Drugs 2009, 69, 1799-1828; (c) Carroll, A. R.; Copp, B. R.; Davis, R. A.; Keyzers, R. A.; Prinsep, M. R. Marine Natural Products. Nat. Prod. Rep. 2020, 37, 175-223.

(2) (a) Williamson, A. Ueber die Theorie der Aetherbildung Justus Liebigs Ann. Chem. 1851, 77, 37-49; (b) Freedman, H. H.; Dubois, R. A. An Improved Williamson Ether Synthesis using Phase Transfer Catalysis. Tetrahedron Lett. 1975, 16, 3251-3254; (c) Kürti, L.; Czakó, B. Strategic applications of named reactions in organic synthesis, 484-485 (Elsevier, 2005)

(3) Zhang, S.-Y.; Zhang, F.-M.; Tu, Y.-Q. Direct Sp $\mathrm{S}^{3} \alpha-\mathrm{C}-\mathrm{H}$ Activation and Functionalization of Alcohol and Ether. Chem. Soc. Rev. 2011, 40, 1937-1949.

(4) (a) Roughley, S. D.; Jordan, A. M. The Medicinal Chemist's Toolbox: An Analysis of Reactions Used in the Pursuit of Drug Candidates. J. Med. Chem. 2011, 54, 3451-3479; (b) Wuitschik, G.; Rogers-Evans, M.; Mueller, K.; Fischer, H.; Wagner, B.; Schuler, F.; Polonchuk, L.; Carreira, E. M. Oxetanes as Promising Modules in Drug Discovery. Angew. Chem. Int. Ed. 2006,45, 77367739 .

(5) Xiang, J.; Shang, M.; Kawamata, Y.; Lundberg, H.; Reisberg, S. H.; Chen, M.; Mykhailiuk, P.; Beutner, G.; Collins, M. R.; Davies, A.; Del Bel, M.; Gallego, G. M.; Spangler, J. E.; Starr, J.; Yang, S.; Blackmond, D. G.; Baran, P. S. Hindered Dialkyl Ether Synthesis with Electrogenerated Carbocations Nature 2019,573, 398402.

(6) Shibutani, S.; Kodo, T.; Takeda, M.; Nagao, K.; Tokunaga, N.; Sasaki, Y.; Ohmiya, H. Organophotoredox-Catalyzed Decarboxylative $\mathrm{C}\left(\mathrm{sp}^{3}\right)-\mathrm{O}$ Bond Formation. J. Am. Chem. Soc. 2020, 142, 1211-1216. 
(7) For further techniques employing the photocatalytic generation of a carbocation intermediate, see: (a) Zhu, Q.; Gentry, E. C.; Knowles, R. R. Catalytic Carbocation Generation Enabled by the Mesolytic Cleavageof Alkoxyamine Radical Cations. Angew. Chem. Int. Ed. 2016, 55, 9969-9973; (b) Webb, E. W.; Park, J. B.; Cole, E. L.; Donnelly, D. J.; Bonacorsi, S. J.; Ewing, W. R.; Doyle, A. G. Nucleophilic (Radio)Fluorination of Redox-Active Esters via Radical Polar Crossover Enabled by Photoredox Catalysis. J. Am. Chem. Soc. 2020, 142, 9493-9500.

(8) Arendt, K. M.; Doyle, A. G. Dialkyl Ether Formation by Nickel-Catalyzed Cross-Coupling of Acetals and Aryl Iodides. Angew. Chem. Int. Ed. 2015, 54, 9876-9880;

(9) Lin, Z.; Lan, Y.; Wang, C. Synthesis of gem-Difluoroalkenes via Nickel-Catalyzed Reductive $\mathrm{C}-\mathrm{F}$ and $\mathrm{C}-\mathrm{O}$ Bond Cleavage. ACS Catal. 2019, 9, 775-780.

(10) Rossolini, T.; Ferko, B.; Dixon, D. J. Photocatalytic Reductive Formation of $\alpha$-Tertiary Ethers from Ketals. Org. Lett. 2019, 21, 6668-6673.

(11) (a) Ohshima, T.; Shibuguchi, T.; Fukuta, Y.; Shibasaki, M. Catalytic Asymmetric Phase-Transfer Reactions Using TartrateDerived Asymmetric Two-Center Organocatalysts. Tetrahedron 2004, 6o, 7743-7754; (b) Yanagisawa, A.; Taga, M.; Astumi, T.; Nishimura, K.; Ando, K.; Taguchi, T.; Tsumuki, H.; Chujo, I.; Mohri, S.-I. Process Development of the $\mathrm{PDE}_{4}$ Inhibitor K-34. Org. Process Res. Dev. 2011, 15, 376-381.

(12) (a) Mukaiyama, T. The Directed Aldol Reaction. Org. React. 1982, 28, 203-331; (b) Kürti, L.; Czakó, B. Strategic applications of named reactions in organic synthesis, 298-299 (Elsevier, 2005)

(13) (a) Saito, I.; Nagata, R.; Yuba, K.; Matsuura, T. Synthesis of $\alpha$-Silyloxyhydroperoxides from the Reaction of Silyl Enol Ethers and Hydrogen Peroxide. Tetrahedron Lett. 1983, 24, 1737-1740; (b) Norris, R. O.; Verbanc, J. J.; Hennion, G. F. Desaturation Products from 1,3,3-Trimethoxybutane. J. Am. Chem. Soc. 1938, 6o, 1159-1161.

(14) (a) Lo, J. C.; Gui, J.; Yabe, Y.; Pan, C.-M.; Baran, P. S. Functionalized Olefin Cross-Coupling to Construct Carbon-Carbon Bonds. Nature 2014, 516, 343-348; (b) Lo, J. C.; Kim, D.; Pan, C.M.; Edwards, J. T.; Yabe, Y.; Gui, J.; Qin, T.; Gutiérrez, S.; Giacoboni, J.; Smith, M. W.; Holland, P. L.; Baran, P. S. FeCatalyzed C-C Bond Construction from Olefins via Radicals. J. Am. Chem. Soc. 2017, 139, 2484-2503; (c) Green, S. A.; Huffman, T. R.; McCourt, R. O.; van der Puyl, V.; Shenvi, R. A. Hydroalkylation of Olefins To Form Quaternary Carbons. J. Am. Chem. Soc. 2019, 141, 7709-7714; (d) Crossley, S. W. M.; Obradors, C.; Martinez, R. M.; Shenvi, R. A. Mn-, Fe-, and Co-Catalyzed Radical Hydrofunctionalizations of Olefins. Chem. Rev. 2016, 116, 89129000 and references therein

(15) (a) Leitch, J. A.; Rossolini, T.; Rogova, T.; Maitland, J. A. P.; Dixon, D. J. $\alpha$-Amino Radicals via Photocatalytic SingleElectron Reduction of Imine Derivatives. ACS Catal. 2020, 10, 2009-2025; (b) Xia, Q.; Dong, J.; Song, H.; Wang, Q. Visible-Light Photocatalysis of the Ketyl Radical Coupling Reaction. Chem. Eur. J. 2019, 25, 2949-2961; (c) Trowbridge, A.; Walton, S. M.; Gaunt, M. J. New Strategies for the Transition-Metal Catalyzed Synthesis of Aliphatic Amines. Chem. Rev. 2020, 120, 2613-2692.

(16) Xie, L.-G.; Rogers, J.; Anastasiou, I.; Leitch, J. A.; Dixon, D. J. Iridium-Catalyzed Reductive Allylation of Esters. Org. Lett. 2019, 21, 6663-6667; (b)

(17) For other seminal papers using $\alpha$-oxy radicals, see: (a) Jeffey, J. L.; Terrett, J. A.; MacMillan, D. W. C. O-H Hydrogen Bonding Promotes $\mathrm{H}$-Atom Transfer from $\alpha \mathrm{C}-\mathrm{H}$ Bonds for CAlkylation of Alcohols. Science 2015, 349, 1532-1536; (b) Jin, J.; MacMillan, D. W. C. Direct $\alpha$-Arylation of Ethers through the Combination of Photoredox-Mediated $\mathrm{C}-\mathrm{H}$ Functionalization and the Minisci Reaction. Angew. Chem. Int. Ed. 2015, 54, 15651569.
(18) (a) Fischer, H.; Radom, L. Factors Controlling the Addition of Carbon-Centered Radicals to Alkenes-An Experimental and Theoretical Perspective. Angew. Chem. Int. Ed. 20o1, 40, 1340-1371; (b) Qin, T.; Malins, L. R.; Edwards, J. T.; Merchant, R. R.; Novak, A. J. E.; Zhong, J. Z.; Mills, R. B.; Yan, M.; Yuan, C.; Eastgate, M. D.; Baran, P. S. Nickel-Catalyzed Barton Decarboxylation and Giese Reactions: A Practical Take on Classic Transforms. Angew. Chem. Int. Ed. 2017, 56, 260-265.

(19) (a) Okimoto, Y.; Sakaguchi, S.; Ishii, Y. Development of a Highly Efficient Catalytic Method for Synthesis of Vinyl Ethers. J. Am. Chem. Soc. 2002, 124, 1590-1591; (b) Nordmann, G.; Buchwald, S. L. A Domino Copper-Catalyzed C-O Coupling-Claisen Rearrangement Process. J. Am. Chem. Soc. 2003, 125, 4978-4979; (c) Dehli, J. R.; Legros, J.; Bolm, C. Synthesis of Enamines, Enol Ethers and Related Compounds by Cross-Coupling Reactions. Chem. Commun. 2005, 973-986.

(20) (a) Cannizzo, L. F.; Grubbs, R. H. In situ preparation of $(\mu$-chloro $)(\mu$ -

methylene)bis(cyclopentadienyl)(dimethylaluminum)titanium (Tebbe's reagent). J. Org. Chem. 1985, 50, 2386-2387; (b) Pine, S. H.; Zahler, R.; Evans, D. A.; Grubbs, R. H. Titanium-Mediated Methylene-Transfer Reactions. Direct Conversion of Esters into Vinyl Ethers. J. Am. Chem. Soc. 1980, 102, 3270-3272; (c) Hartley, R. C.; McKiernan, G. J. Titanium reagents for the alkylidenation of carboxylic acid and carbonic acid derivatives. J. Chem. Soc., Perkin Trans. 1, 2002, 2763-2793.

(21) Accordingly, trace $\mathrm{HCl}$ in $\mathrm{TMSCl}$ is unlikely to be of mechanistic relevance.

(22) Synthesis of HE-Ph on $>100 \mathrm{~g}$ scale is detailed in the ESI.

(23) Aycock, R. A.; Vogt, D. B.; Jui, N. T. A Practical and Scalable System for Heteroaryl Amino Acid Synthesis. Chem. Sci. 2017, 8, 7998-8003.

(24) Using DMF- $d_{7}$ as the solvent resulted in no deuterium incorporation into the product at any position.

(25) Whitaker, K. S.; Whitaker, D. T. 2-Methoxypropene. Encyclopedia of Reagents for Organic Synthesis, 1-3 (2001) DOI: 10.1002/047084289x.rm124.

(26) The nature of this promotion remains unclear. ${ }^{1} \mathrm{H}$ NMR experiments show no ground-state interactions between HE and TMSCl. However, that does not rule out an interaction between the TMSCl and a downstream oxidized form of the HE, see: (a) Gimeno, M. C.; Herrera, R. P. Hydrogen Bonding and Internal or External Lewis or Brønsted Acid Assisted (Thio)urea Catalysts. Eur. J. Org. Chem. 2020, 2020, 1057-1068; (b) Ishihara, K.; Kaneeda, M.; Yamamoto, H. Lewis Acid Assisted Chiral Bronsted Acid for Enantioselective Protonation of Silyl Enol Ethers and Ketene Bis(trialkylsilyl) Acetals. J. Am. Chem. Soc. 1994, 116, 11179-11180; (c) Ishihara, K.; Nakamura, S.; Kaneeda, M.; Yamamoto, H. First Example of a Highly Enantioselective Catalytic Protonation of Silyl Enol Ethers Using a Novel Lewis Acid-Assisted Brønsted Acid System. J. Am. Chem. Soc. 1996, 118, 12854-12855; (d) Ishihara, K.; Kaneeda, M.; Yamamoto, H. Lewis Acid Assisted Chiral Brønsted Acid for Enantioselective Protonation of Silyl Enol Ethers and Ketene Bis(trialkylsilyl) Acetals. J. Am. Chem. Soc. 1994, 116, 11179-11180.

(27) (a) Reisman, S. E.; Doyle, A. G.; Jacobsen, E. N. Enantioselective Thiourea-Catalyzed Additions to Oxocarbenium Ions. J. Am. Chem. Soc. 20o8, 130, 7198-9199; (b) Ford, D. D.; Lehnherr, D.; Kennedy, C. R.; Jacobsen, E. N. Anion-Abstraction Catalysis: The Cooperative Mechanism of $\alpha$-Chloroether Activation by Dual Hydrogen-Bond Donors. ACS Catalysis 2016, 6, 4616-462o.

(28) (a) Trowbridge, A.; Reich, D.; Gaunt, M. J. Multicomponent Synthesis of Tertiary Alkylamines. Nature 2018, 561, 522527; (b) Wang, P.-Z.; Chen, J.-R.; Xiao, W.-J. Hantzsch Esters: an Emerging Versatile Class of Reagents in Photoredox Catalyzed Organic Synthesis. Org. Biomol. Chem. 2019, 17, 6936-6951. 
(29) A mechanism involving single electron oxidation of the enol ether $\left(E_{1 / 2}^{\circ}=+1.19 \mathrm{~V}\right)$ is ruled out as: (i) the Hantzsch ester has a substantially lower oxidation potential $\left(E_{1 / 2}^{\circ}=+0.75 \mathrm{~V}\right)$, (ii) photocatalysts such as $\left[\operatorname{Ir}(\mathrm{ppy})_{2}(\mathrm{dtbbpy})\right] \mathrm{PF}_{6}$ - which are substantially less oxidizing (unable to be reduced by enol ether 1a) still lead to $62 \%$ conversion to $3 a$.

(30) Previous studies demonstrate luminescence quenching of the iridium photocatalyst by similar Hantzsch esters, see: Leitch, J. A.; Rogova, T.; Duarte, F.; Dixon, D. J. Dearomative Photocatalytic Construction of Bridged 1,3-Diazepanes. Angew. Chem. Int. Ed. 2020, 59, 4121-4130.
(31) De Vleeschouwer, F.; Van Speybroeck, V.; Waroquier, M.; Geerlings, P.; De Proft, F. Electrophilicity and Nucleophilicity Index for Radicals. Org. Lett. 2007, 9, 2721-2724.

(32) (a) Caron, A.; Morin, E.; Collins, S. K. Bifunctional Copper-Based Photocatalyst for Reductive Pinacol-Type Couplings. ACS Catal. 2019, 9, 9458-9464; (b) Reich, D.; Trowbridge, A.; Gaunt, M. J. Rapid Syntheses of (-)-FR901483 and (+)-TAN1251C Enabled by Complexity-Generating Photocatalytic Olefin Hydroaminoalkylation. Angew. Chem. Int. Ed. 2020, 59, 2256-2261.

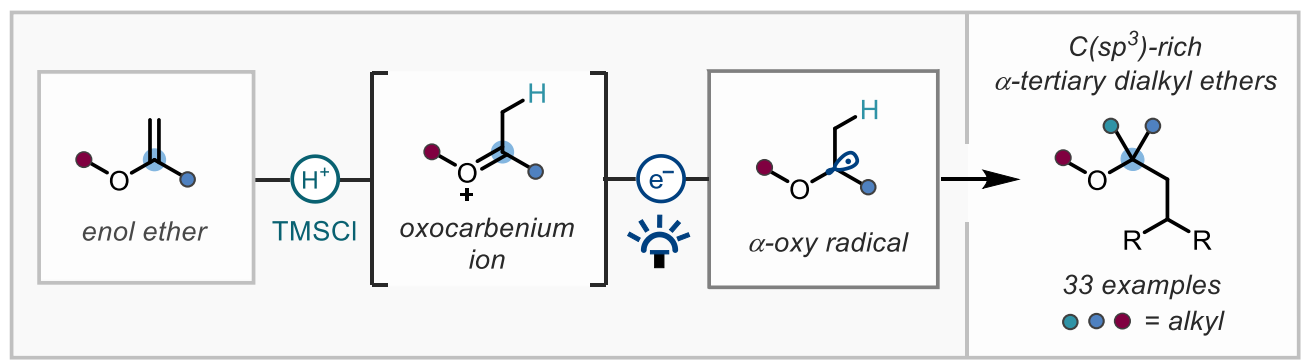

